\title{
CFAR DETECTION IN MIMO RADARS USING FUZZY FUSION RULES IN HOMOGENEOUS BACKGROUND
}

\author{
Faycal Khaldi ${ }^{1}$ and Faouzi Soltani ${ }^{2}$ \\ ${ }^{1,2}$ Département d'électronique, Université des Frères Mentouri Constantine \\ Constantine 25000, Algeria
}

\begin{abstract}
In this paper, we propose to use fuzzy fusion rules to improve the performances of the Cell Averaging Constant False Alarm Rate (CA-CFAR) detector for MIMO (Multiple Input Multiple Output) radars in homogenous background modeled by a Pareto distribution. We compute the membership function for each individual detector. The global membership function at the fusion centre is a combination of the membership functions collected from individual detectors using four fusion rules, namely; the "MIN", "MAX", "algebraic product" and the "algebraic sum". By means of Monte Carlo simulations, we evaluated the performance of the global system. The obtained results showed that for a number of nodes equal to four, the performance is the best for a high number of receivers and a low number of transmitters. For the best case and in homogenous background, the "algebraic product" fusion rule gives the best result when SNR $>4 d B$ whereas the "algebraic sum" is the best when SNR $<4 d B$ for the CA-CFAR.
\end{abstract}

\section{KEYWORDS}

Radar detection, MIMO, Fuzzy rules, Homogeneous clutter, CFAR

\section{INTRODUCTION}

A MIMO radar structure is a multi-antenna radar system which consists of using multiple antennas at the transmitter and the receiver. It is therefore a generalization of the concept of multi-static radars [1].

This technique was first used in communications and has recently been extended to radars where the received signals are jointly processed at the multiple receiving antennas. It has the advantage of improving radar performance, in terms of the regulation of the false alarm rate and the maximization of the probability of detection [2]. MIMO radars are, generally divided into two types; the coherent MIMO radar "co-located" and the statistical MIMO radar "widely separated". The statistical MIMO radar takes advantage of the spatial diversity in the transmit and receive angles to enhance the detection performance.

The difference between the two configurations is the signal model: For Widely Separated antennas, the spatial properties of extended targets are exploited while the target is modelled as a point with no spatial property for coherent MIMO radars [3]. 
In radar automatic detection system, the main goal is to design an algorithm that set the threshold adaptively to deal with the changing power of the clutter and to keep the false alarm rate constant and at a desired value.

The performances of the CA-CFAR approaches those of the optimum detector if the reference cells are Gaussian, independent and identically distributed (iid).
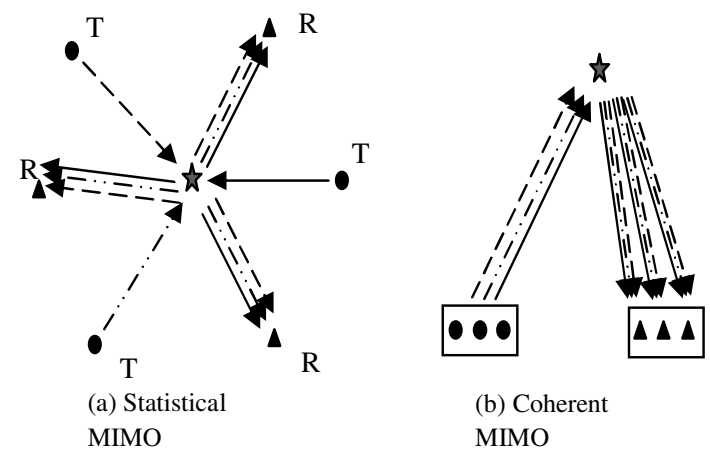

(b) Coherent MIMO

Figure. 1 MIMO radar concept.

In practical situations, the clutter may not be homogeneous. This non homogeneity is caused by either the presence of interfering targets or an extended clutter edge. In this case, the performance of the CA-CFAR degrades seriously. In order to overcome this problem, several modified versions of the CA-CFAR detector were proposed and are generally based on the order statistic technique: the OS-CFAR [4], the GOSCA-CFAR [5], the OSGO-CFAR and the OSSO-CFAR [6].

Several works dealing with target detection in MIMO radars in the Neyman-Pearson sense [7, 8] or using the Generalized Likelihood Ratio [9] have been reported in the literature.

In [10], Janatian generalized the CA-CFAR, the SO-CFAR, the OS-CFAR and the ACMLD (Automatic Censored Mean-Level Detector) for a Gaussian background. Widely Separated MIMO radars were used in homogeneous and non-homogeneous clutter (presence of interfering targets).

Using INGARA database, Weinberg [11] showed that the Pareto distribution offers a good fitting performance as a model for high-resolution high-grazing angle sea clutter.

This paper deals with the analysis of the performance of Widely Separated MIMO radars where the individual detectors are the CA-CFAR detector. Instead of using binary fusion rules, we propose to extend this technique to the use fuzzy fusion rules at the fusion centre. The clutter is assumed to follow a Pareto distribution.

The rest of the paper is organized as follow: section 2 describes the signal model in MIMO radars. In section 3, we analyze the fuzzy CA-CFAR detector in MIMO radars in a Pareto clutter. The results concerning the performance of the MIMO structure in homogeneous clutter are presented and commented in section 4 and followed by some concluding remarks in section 5 . 


\section{SIGNAL MODEL IN MIMO RADARS}

The structure under consideration is a MIMO radar system that has $\mathrm{M}$ transmit antennas and $\mathrm{N}$ receive antennas. The antennas are assumed to be widely separated as shown in Fig. 1.a. It is also assumed that the $\mathrm{m}^{\text {th }}$ transmitter delivers a signal $\sqrt{E / M} s_{m}(t)$, where $\mathrm{E}$ is the total transmitted power and $\left\|s_{m}(t)\right\|^{2}=1$. That is, systems with a reduced number of nodes (couples Tx-Rx) have an increased available power per node. In other words, each of the $M$ transmitters provides a power equal to $\frac{E}{M}$. The $\mathrm{n}^{\text {th }}$ received signal is modelled as follows:

$$
r_{n}(t)=\sum_{m-1}^{M} \alpha_{m, n}(\sigma) s_{m}\left(t-\frac{R_{m, n}}{c}\right)+e_{n}(t)
$$

Where $s_{m}(t)$ is the $m^{\text {th }}$ transmitted signal, $e(t)$ is an additive thermal noise, $\alpha_{m, n}$ is a complex coefficient including the amplitude and the phase of the received signal and is given by the following expression.

$$
\sqrt{\frac{E}{M}} \sqrt{\frac{G_{t} G_{r} \lambda_{m}^{2} \sigma}{(4 \pi)^{3} R_{m}^{2} R_{n}^{2}}} \exp \left(-j \frac{2 \pi R_{m, n}}{\lambda_{m}}\right) .
$$

If the transmitted waveforms are assumed to be orthogonal in such a way that they can be separated in each receiver, the received signal after the matched filtering can be expressed as:

$$
q_{m, n}=\alpha_{m, n}+n_{m, n} .
$$

The MIMO radar detection problem can be formulated in terms of the binary hypothesis test as follow:

$$
Q_{0}=\left\{\begin{array}{ll}
n, & H_{0} \\
\alpha+n, & H_{1}
\end{array} .\right.
$$

Under hypothesis $\mathrm{H}_{0}$, the target is declared absent in the received signal and the signal is constituted of clutter only. Under hypothesis $\mathrm{H}_{1}$, a is target declared present and the received signal is the sum of both target and clutter signals.

The classical Neyman-Pearson detector uses the Likelihood ratio test and is given by:

$$
T\left(q_{0}\right)=\log \frac{P\left(q_{0} / H_{1}\right)}{P\left(q_{0} / H_{0}\right)} \gtrless \gamma .
$$

Where $P\left(q_{0} \mid H_{l}\right)$ and $P\left(q_{0} \mid H_{0}\right)$ are the probability density functions of the observation vector under the hypothesis $\mathrm{H}_{1}$ or $\mathrm{H}_{0}$ respectively. The threshold $\gamma$ is determined by a prescribed probability of false alarm.

The Likelihood ratio test in (4) is equivalent to the following test [2]:

$$
\left\|Q_{0}\right\|^{2} \gtrless \gamma
$$

Where $Q_{0}$ is the matched filter output of the cell under test (CUT). For a CA-CFAR detector, $\left\|Q_{0}\right\|^{2}$ is the sum of the CUT powers, $\gamma=\mathrm{T}^{*} \mathrm{Z}$ is the detection threshold, $\mathrm{Z}$ is the noise power level estimation and $\mathrm{T}$ is a factor that adjusts the $P_{f a}$ at a desired value. 
The structure of the received data is shown in Fig. 2. The range gating method is employed in only one angular-resolution cell (Fig. 2.a). Each receiver can sense the echoes of all transmitters and therefore, this structure can be formulated as a matrix with $\mathrm{M}^{*} \mathrm{~N}$ columns and each column contains a CUT (grey cells) plus $2 *$ L reference cells as shown in Fig. 2.b.

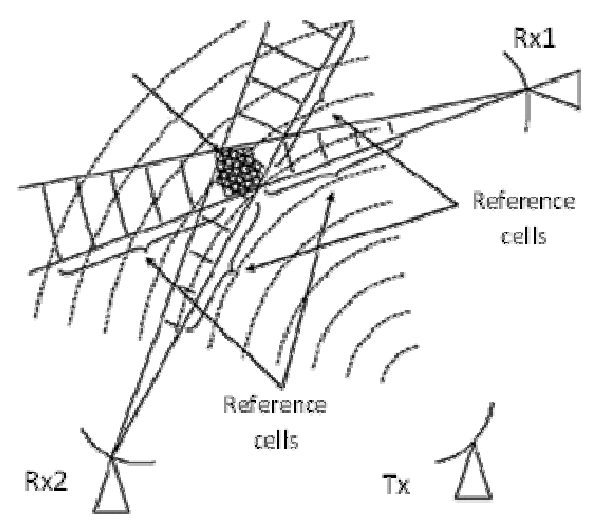

(a) MIMO radar resolution cells

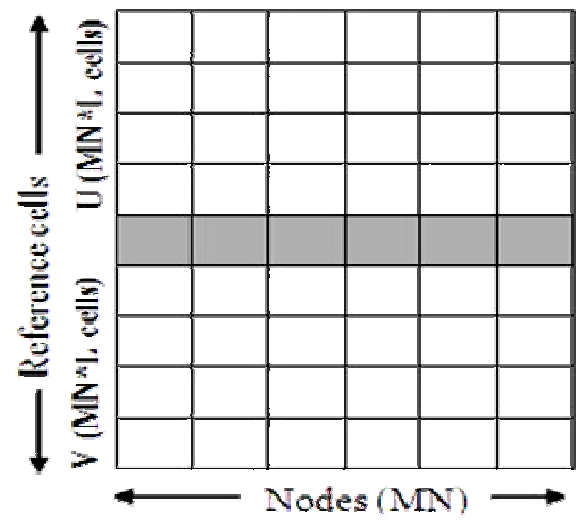

(b) MIMO radar simplified configuration

Figure 2 MIMO Radar Concept

\section{FUZZY CA-CFAR DETECTOR FOR MIMO RADARS}

\subsection{Fuzzy CA-CFAR Detector in Pareto Clutter}

A block diagram of the fuzzy CA-CFAR detector under consideration is shown in Fig. 3.The received signal is sampled in range by the range resolution cells. The statistics which is the membership function is compared to a threshold to decide about the presence or the absence of a target.

The clutter is assumed to be modeled by a Pareto distribution which is defined as follows:

$$
f_{X}(x)=\frac{\alpha \beta^{\alpha}}{x^{\alpha+1}}
$$

Where $\alpha$ is the shape parameter and $\beta$ the scale parameter.

In the CA-CFAR detector, the membership function to the false alarm space is given by [12 ] 
$\mu(z)=P\left(R>z / H_{0}\right)$

Where

$R=\frac{Q_{0}}{Y}$
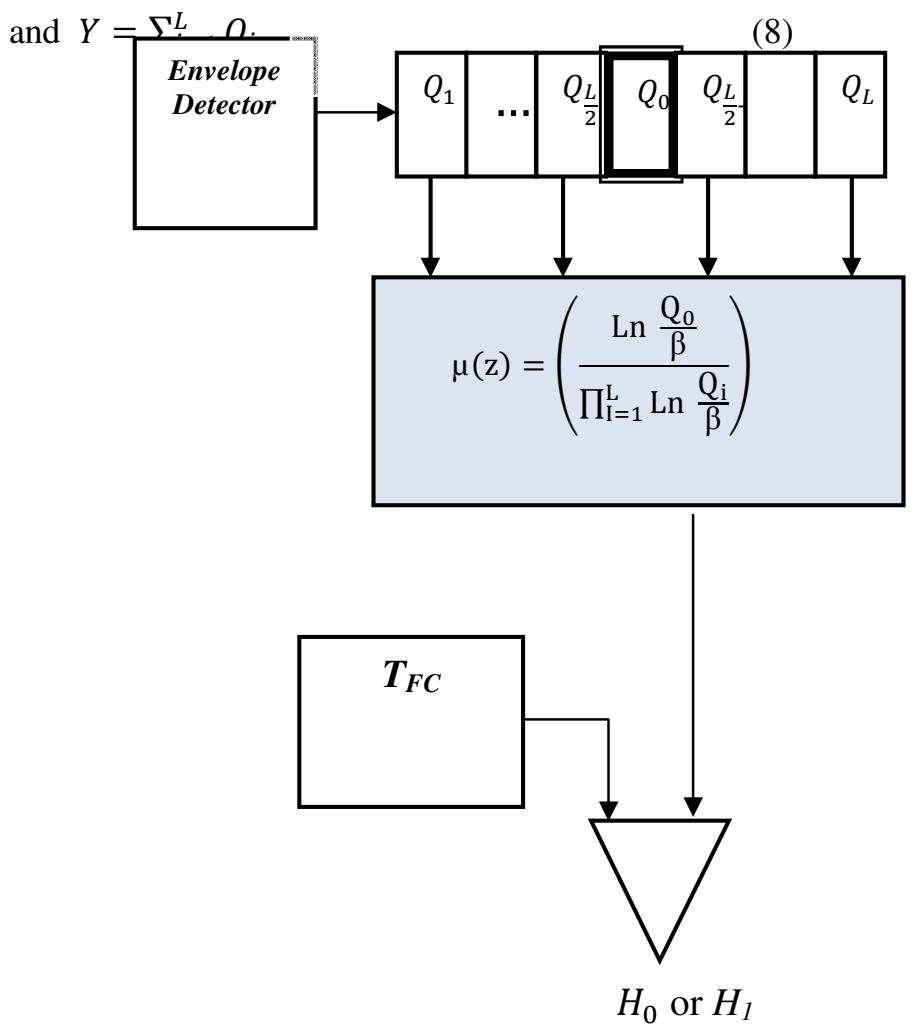

Figure. 3 Block Diagram of the fuzzy CA-CFAR detector

Since the clutter obeys to a Pareto distribution, it was shown that the following transformation leads to an exponential distribution

$$
Q_{i} \simeq \beta e^{X i}
$$

Where $Q_{i}$ follows a Pareto distribution with parameters $\alpha$ and $\beta, Q_{i} \simeq P a(\alpha, \beta)$ and $\mathrm{X}_{\mathrm{i}}$ follows an exponential distribution with parameter $\alpha, X_{i} \simeq \exp (\alpha)$

The decision rule concerning a fuzzy CA-CFAR detector is given by [ ]

$$
\mu: X \rightarrow\left\{\begin{array}{cc}
1 & \frac{X_{0}}{\sum_{i=1}^{L} X_{i}}>T \\
0 & \frac{X_{0}}{\sum_{i=1}^{L} X_{i}}<T
\end{array}\right.
$$

Using the transformation in (9), the decision rule becomes

$$
\mu: Q \rightarrow \begin{cases}1 & \frac{Q_{0}}{\prod_{i=1}^{L} Q_{i}}>T \cdot \beta^{1-L} \\ 0 & \frac{Q_{0}}{\prod_{i=1}^{L} Q_{i}}<T \cdot \beta^{1-L}\end{cases}
$$


The membership function to the false alarm space is then

$$
\mu(z)=\operatorname{Prob}\left(\frac{\operatorname{Ln}\left(\frac{Q_{0}}{\beta}\right)}{\prod_{i=1}^{L} \operatorname{Ln}\left(\frac{Q_{i}}{\beta}\right)}>T / H_{0}\right)
$$

Using the results in [12], the membership function reduces to

$$
\mu(z)=\frac{1}{(z+1)^{L}}
$$

\subsection{Fuzzy Fusion Rules in MIMO Radars}

The number of fuzzy decisions $\left(D_{1}, D_{2}, \ldots, D_{M N}\right)$ is equal to the number of nodes M.N where $M$ is the number of transmitters and $\mathrm{N}$ is the number of receivers. Each node transmits its membership function to the fusion centre where a global membership function $\mu_{F C}$ is derived. The fuzzy fusion rules considered in this work are "MIN", "MAX", "Algebraic sum" and "Algebraic product".

1) The "MIN" fusion rule

The membership function at the fusion centre, $\mu_{F C}$ is defined as

$$
\mu_{F C}=\operatorname{MIN}\left(\mu_{D_{1}}, \mu_{D_{2}}, \ldots . \mu_{D_{M N}}\right)
$$

The corresponding threshold at the fusion centre is [12]

$$
T_{F C}=1-(1-P f a)^{\frac{1}{M N}}
$$

2) The "MAX" fusion rule

For this rule, $\mu_{F C}$ is defined as

$$
\mu_{F C}=\operatorname{MAX}\left(\mu_{D_{1}}, \mu_{D_{2}}, \ldots . \mu_{D_{M N}}\right)
$$

The corresponding threshold at the fusion centre is [12]

$$
T_{F C}=P f a^{\frac{1}{M N}}
$$

3) The "Algebraic sum" fusion rule

Following the same reasoning as previously, $\mu_{F C}$ is found to be

$$
\mu_{F C}=1-\prod_{i=1}^{M N}\left(1-\mu_{D_{i}}\right)
$$

The corresponding threshold at the fusion centre is obtained from the Pfa expression [13]

$$
P f a=1-\frac{\Gamma\left(\mathrm{MN},-\mathrm{Ln}\left(1-\mathrm{T}_{\mathrm{FC}}\right)\right)}{(M N-1) !}
$$


4) The "Algebraic product" fusion rule

In this case, $\mu_{F C}$ is defined as

$$
\mu_{F C}=\prod_{i=1}^{M N}\left(1-\mu_{D_{i}}\right)
$$

The corresponding threshold at the fusion centre is obtained from the Pfa expression [13]

$$
P f a=\frac{\Gamma\left(\mathrm{MN},-\mathrm{Ln}\left(1-\mathrm{T}_{\mathrm{FC}}\right)\right)}{(M N-1) !}
$$

\section{RESULTS AND DISCUSSIONS}

To demonstrate the effectiveness of using fuzzy fusion rules in a MIMO structure, we used Monte-Carlo simulations to assess the detection probability $\left(P_{D}\right)$ as a function of the signal-toclutter ratio (SCR). We assume that the number of reference cells is $\mathrm{L}=16$, the design $P_{f a}$ is equal to $10^{-4}$ and the clutter is homogeneous. We also assume that the number of nodes $\mathrm{MN}$ is equal to 4 with three different cases $(\mathrm{M}=4$ and $\mathrm{N}=1, \mathrm{M}=2$ and $\mathrm{N}=2, \mathrm{M}=1$ and $\mathrm{N}=4)$. For the four fusion rules considered, the thresholds at the fusion centre are calculated using expressions (15), (17), (19) and (21).

Fig. 4 shows the detection performance with respect to $\mathrm{M}$ and $\mathrm{N}$. It is clear that the highest $P_{D}$ is obtained for $\mathrm{M}=1$ and $\mathrm{N}=2$. In general, the performance increases as the number of receivers increases.

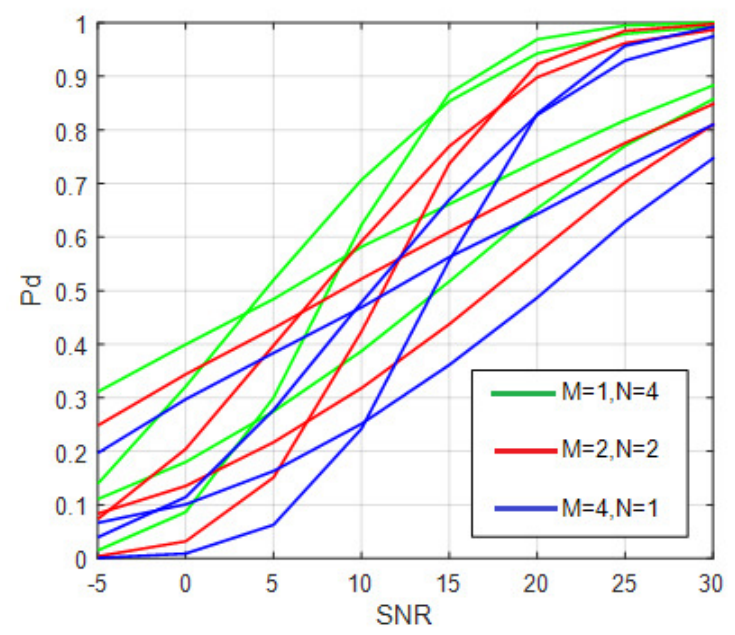

Figure. 4 CA-CFAR in MIMO radars with "MIN", "MAX", "algebraic product" and "algebraic sum" fuzzy fusion rules in homogenous background in cases $(M=1, N=4),(M=2, N=2)$ and $(M=4, N=1)$

Next, we compare the performance of the for fusion rules for the case of $M=1$ and $N=4$. We observe that the "Algebraic product "fusion rule gives the best results for an SCR $>4 \mathrm{~dB}$ and the "MIN" fusion rule has comparable performance as the latter SCR > $15 \mathrm{~dB}$. 


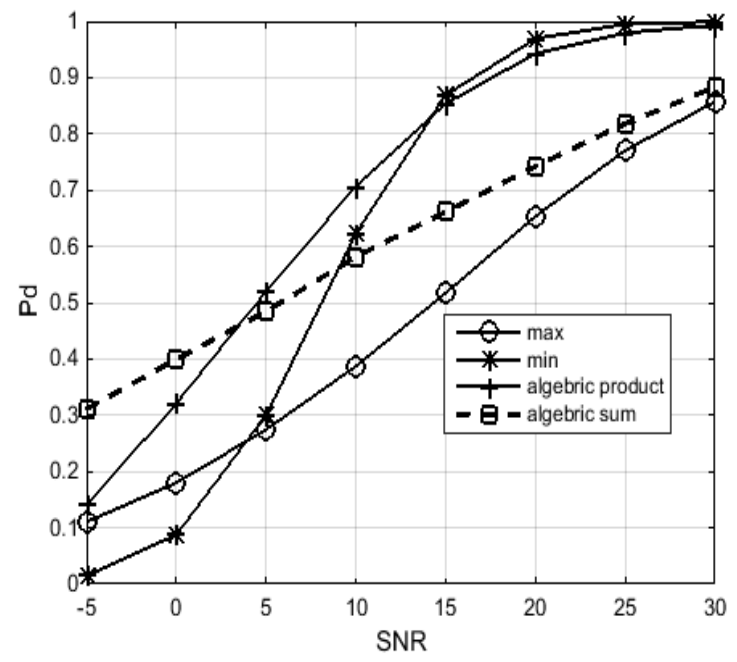

Figure. 5 CA-CFAR in MIMO radars with "MIN", "MAX", "algebraic sum" and "algebraic product" fusion rules in homogenous background

\section{CONCLUSIONS}

In this paper, we evaluated through Monte-Carlo simulations, the performance of a MIMO radar where each detector is constituted of a fuzzy CA-CFAR detector. At the fusion centre, the membership function to the false alarm space from all detectors are received and combined according to four fuzzy fusion rules. Different values of the number of transmitters and receivers were considered. The simulation results demonstrated clearly that the highest probability of detection is attained when the number of receivers increases. In addition, the best performance was provided by the "Algebraic product" fuzzy fusion rule and the "MIN" fusion rule for high SCR.

\section{REFERENCES}

[1] J. Li and P. Stoica, MIMO Radar Signal Processing, Wiley, New Jersey, 2009.

[2] E. Fishler, et al., "Spatial diversity in radars- models and detection performance," IEEE Transaction on Signal Processing, vol. 54, no. 3, pp. 823-838, 2006.

[3] C. Y. Chong, "Signal Processing for MIMO Radars: Detection under Gaussian and non-Gaussian environments and application to STAP," Ph.D. thesis, Supelec, France, 2011.

[4] H. Rohling, "Radar CFAR thresholding in clutter and multiple target situations," IEEE Transactions on Aerospace and Electronic Systems, vol. 19, no. 4, pp. 608-621, 1983.

[5] H. You, "Performance of some generalized modified order statistics CFAR detectors with automatic censoring technique in multiple target situations," IEE Proceedings, Radar, Sonar and Navigation, vol. 141, no. 4, pp. 205-212, 1994.

[6] A.R. Elias-Fuste, M.G. DE Mercado, and E.R. Davo, "Analysis of some modified order statistics CFAR: OSGO and OSSO CFAR," IEEE Transaction on Aerospace and Electronic Systems, vol. 26, no. 1, pp. 197-202, 1990. 
[7] C. Y. Chong, F. Pascal, J.P. Ovarlez, and M. Lesturgie, "MIMO Radar Detection in Non-Gaussian and Heterogeneous Clutter," IEEE Journal of Selected Topics in Signal Processing, vol. 4, no. 1, pp. 115-126, 2010.

[8] G. Cui, L. Kong, and X. Yang, "Multiple-input multiple-output radar detectors design in nonGaussian clutter," IET Radar, Sonar and Navigation, vol. 4, no. 5, pp.724-732, 2010.

[9] J. Liu, Z. J. Zhang, Y. Cao, and S. Yang, “A closed-form expression for false alarm rate of adaptive MIMO-GLRT detector with distributed MIMO radar," Signal Processing, vol. 93, pp.2771-2776, 2013.

[10] N. Janatian, M. Modarres-Hashemi, and A. Sheikhi, "CFAR Detectors for MIMO Radars," Circuits Systems and Signal Processing, vol. 32, no. 3, pp. 1389-1418, 2013.

[11] G.V. Weinberg, 'Assessing Pareto fit to high resolution high grazing angle sea clutter', Electron. Lett., 2011, 47, (8), pp. 516-517.

[12] Z. Hammoudi, F. Soltani, Distributed CA-CFAR and OS-CFAR detection using fuzzy space and fuzzy fusion rules, IEE Proceedings Part F Vol. 151 (3) (2004) 135-142.

[13] H. A. Meziani, F. Soltani, Decentralized Fuzzy CFAR Detector in Homogeneous Pearson Clutter Background, Signal Processing Elsevier, Vol. 91 (2011), 2530-2540.

\section{AUTHORS}

Faouzi Soltani was born in Constantine Algeria in October 1962. He received the Engineer degree from Algiers National Polytechnic in 1985, the MPhil (Eng) degree from Birmingham University (UK) in 1989 and the Doctorat d'état degree from Constantine University in 1999 all in Electronic Engineering. He joined the department of Electronic Engineering of Constantine University in 1989 as an assistant professor then as a full professor in 2004. He is the head of "Signals and Communication Systems“ research laboratory. His main research interests include radar CFAR detection, estimation theory, neural networks and clutter modeling.

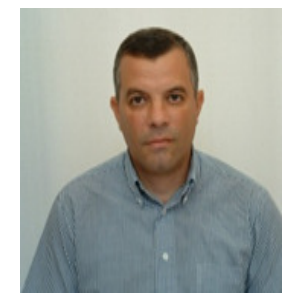

Faycal Khaldi received his Master degree from Medea University, Algeria in 2013. He joined Constantine university as a $\mathrm{PhD}$ student where he is working on MIMO radar signal processing in homogeneous and non homogeneous clutter. 